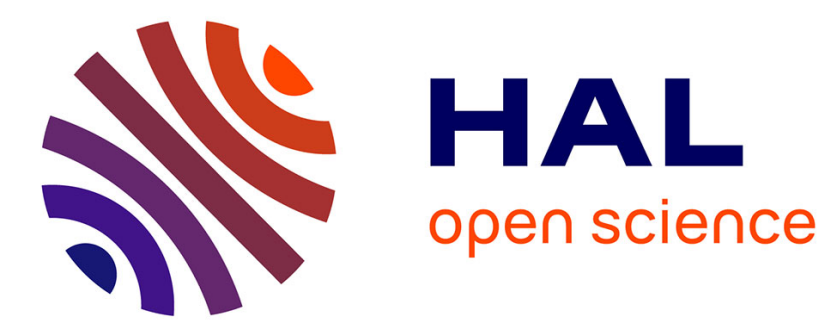

\title{
Mucoadhesive Nanoparticles for Oral Delivery of Insulin
}

\author{
S. Sajeesh, Chandra P Sharma, Christine Vauthier
}

\section{To cite this version:}

S. Sajeesh, Chandra P Sharma, Christine Vauthier. Mucoadhesive Nanoparticles for Oral Delivery of Insulin. Lan-Anh Le; Ross J. Hunter; Victor R. Preedy. Nanotechnology and Nanomedicine in Diabetes, CRC Press, pp.165-185, 2012, 9780429087134. 10.1201/b11775-13 . hal-03196166

\section{HAL Id: hal-03196166 https://hal.science/hal-03196166}

Submitted on 12 Apr 2021

HAL is a multi-disciplinary open access archive for the deposit and dissemination of scientific research documents, whether they are published or not. The documents may come from teaching and research institutions in France or abroad, or from public or private research centers.
L'archive ouverte pluridisciplinaire $\mathbf{H A L}$, est destinée au dépôt et à la diffusion de documents scientifiques de niveau recherche, publiés ou non, émanant des établissements d'enseignement et de recherche français ou étrangers, des laboratoires publics ou privés. 


\title{
Mucoadhesive Nanoparticles for Oral Delivery of Insulin
}

\author{
SAJEESH S. ${ }^{\text {\# }}$, CHANDRA P. SHARMA ${ }^{1}$ and CHRISTINE VAUTHIER ${ }^{2 *}$
}

${ }^{1}$ Division of Biosurface Technology Biomedical Technology Wing Sree Chitra Tirunal Institute for Medical Sciences \& Technology (SCTIMST), Thiruvanathapuram, Kerala, India

${ }^{2}$ Univ. Paris Sud, Physico-chimie, Pharmacotechnie, Biopharmacy, UMR 8612, Chatenay-Malabry, 92 296, France

Published in: In "Nanotechnology and Nanomedicine in Diabetes, 1st Edition." L.A. Le, R.J.Hunter, V.R.Preedy, Eds. CRC Press, Taylor and Francis Group, Boca Raton, FL, USA, Chap 9, pp.165-185 (2012) ISBN 978-1-57808-729-7, eBook ISBN: 9780429087134. Available on https://doi.org/10.1201/b11775.

\#Present address

Nano-Biomaterials Lab Department of Biological Sciences Korea Advanced Institute for Science \& Technology (KAIST), Daejeon 305-701, Republic of Korea

\section{* Corresponding author}

Christine Vauthier,

Univ. Paris Sud, Physico-chimie, Pharmacotechnie, Biopharmacie

UMR CNRS 8612, 92296 CHATENAY-MALABRY Cedex, France

E-mail : Christine.vauthier@u-psud.fr

Phone: 33146835603 Fax 33146835312. 


\begin{abstract}
Insulin was introduced into clinical practice of diabetes treatments soon after it's discovery in 1921. It is still the most effective and safe treatment option available. However, oral insulinotherapy remains a distant reality for diabetic patients, mainly because it is degraded in the gastro-intestinal tract and it is not absorbed under its active form by the gut. Applications of technologies from the nanomedicine represent an opportunity to bypass the bottle neck of the oral administration of insulin and more generally for peptide and protein drugs. Polymeric nanoparticles seem to be the most promising candidate for oral insulin delivery. They are considered as part of the relevant technologies of nanomedicine to achieve oral delivery of insulin. Firstly, they were shown to protect peptides from degradation in the harsh conditions of the gastro-intestinal tract. Secondly, because of their very small size they are believed to have a high potential to enhance drug transport across the barriers found in the gastro-intestinal tract. Aparts form the chemical and biochemical barriers, one of the physical barriers is the mucus that needs to be crossed before absorption sites can be reached on the surface of the epithelium formed by a monolayer of cells. This chapter focuses on the mucoadhesive properties of polymer nanoparticles. These properties are important to consider for the success of nanomedicine in oral delivery of insulin as they will give the formulation suitable properties to reach absorption sites. In a first part, mucoadhesive polymers are presented with their mode of interactions with mucus prior to their use to design mucoadhesive nanoparticles. The second part presents the different mechanisms and modes of action of mucoadhesive nanoparticles that are discussed considering examples from the literature.
\end{abstract}

\author{
Abbreviations \\ AUC: area under the curve \\ CS : chitosan \\ GIT : gastro intestinal tract \\ HPMCP : hydroxypropyl methyl cellulose phtalate \\ PAA : polyacrylic acid \\ PCP: poly(methacrylic acid)-chitosan-poly(ethylene glycol) \\ PEG : poly(ethylene glycol) \\ PFASA: poly(fumaric-co-sebacic acid) \\ PLGA : poly(lactic-co-glycolic acid) \\ PMAA : polymethacrylic acid \\ TMC: trimethylchitosan \\ WGA: wheat germ agglutinin
}




\section{Introduction}

Insulin is used in therapy of both type I and type II diabetes. It is the only efficient treatment for type I diabetes. As most protein pharmaceuticals, insulin are currently delivered by invasive routes of administration including subcutaneous injections (Ramezan and Sharma 2009). The need to find a non-injectable form (i.e.: non-invasive route of administration) has focused on the oral route (Ramezan and Sharma 2009). Apart from safety and patient compliance, oral administration of insulin is of clinical relevance for the treatment of diabetes (Arbit 2004). Indeed, in a healthy human, physiologically secreted insulin from pancreas enters portal circulation first and inhibits the hepatic glucose production. In this process, insulin undergoes a metabolism in the liver to a significant extent (more than 50\%) and thereby the excess glucose is converted and stored in the form of glycogen. Thereafter, insulin not used by the liver finds its way to the peripheral circulation. This mechanism reduces the chance of hypoglycemic effect. This is contrast with the distribution of exogenous insulin obtained after subcutaneous injection to diabetic patients. In this case, insulin distributes first in the peripheral circulation increasing risks of hypoglycemic effects and being ineffective to deliver the required amount of insulin to the liver to maintain their normal physiological function in glucose metabolism (Arbit 2004). Although oral administration of insulin is indisputably the best route of administration, all efforts put to develop suitable delivery formulation since its discovery remained unsuccessful because peptides and protein drugs are degraded in digestive media and are not absorbed as intact molecules by the intestinal epithelium (Lee and Yamamoto 1989). On the basis of the physiology, challenges to oral delivery of peptide and protein drugs include enzymatic degradation and poor permeation across the gastrointestinal tract (GIT) (Lee and Yamamoto 1989).

Oral administration of insulin required a suitable formulation ensuring protection against degradation and helping absorption of the entire protein. Nanomedicine includes 
emerging therapeutical approaches taking advantages of the small size of material used as drug carriers to help drugs to bypass biological barriers. It is a very active field of research on the oral delivery of insulin problem (Ramesan and Sharma 2009) . Association of insulin with formulations used in nanomedicine improved dramatically the resistance of the hormone against degradation by proteases (Madsen and Peppas 1999). This is in favor to the success of the method as the slow rate of degradation may enhance the amount of proteins available for absorption across the GIT. However, insulin absorption by the epithelium still remains a limitation to the development of oral insulino-therapy (Ramazan and Sharma 2009). To achieve the expected benefit from nanomedicine, its necessary to develop nano-systems by optimizing their mucoadhesive properties and giving them other functionalities such as permeation enhancing and protease inhibition properties. The present chapter was aimed to summarize the different approaches used to improve mucoadhesive properties of polymer nanoparticles. The first part presents polymers which were used as mucoadhesives in pharmaceutical formulations. The second part summarizes works done on mucoadhesive nanoparticles including those devoted to improve oral delivery of insulin. Transport and translocation of nanoparticles through the epithelium is the subject of another chapter of this book by Woitiski and will not be discussed in the present chapter.

\section{Mucoadhesion.}

The term 'mucoadhesion' refers to adhesion between polymeric material and mucosal surfaces. Polymers, with certain structural features, become adhesive to mucus layer upon hydration. Though exact reason for adhesion may vary with the types of polymer, formulations containing mucoadhesive polymers are interesting because they provide intimate contact with the mucosal layer and thereby introduce drugs directly on the top of the enterocytes responsible for the highly selective permeability of the gut (Figure 1). 


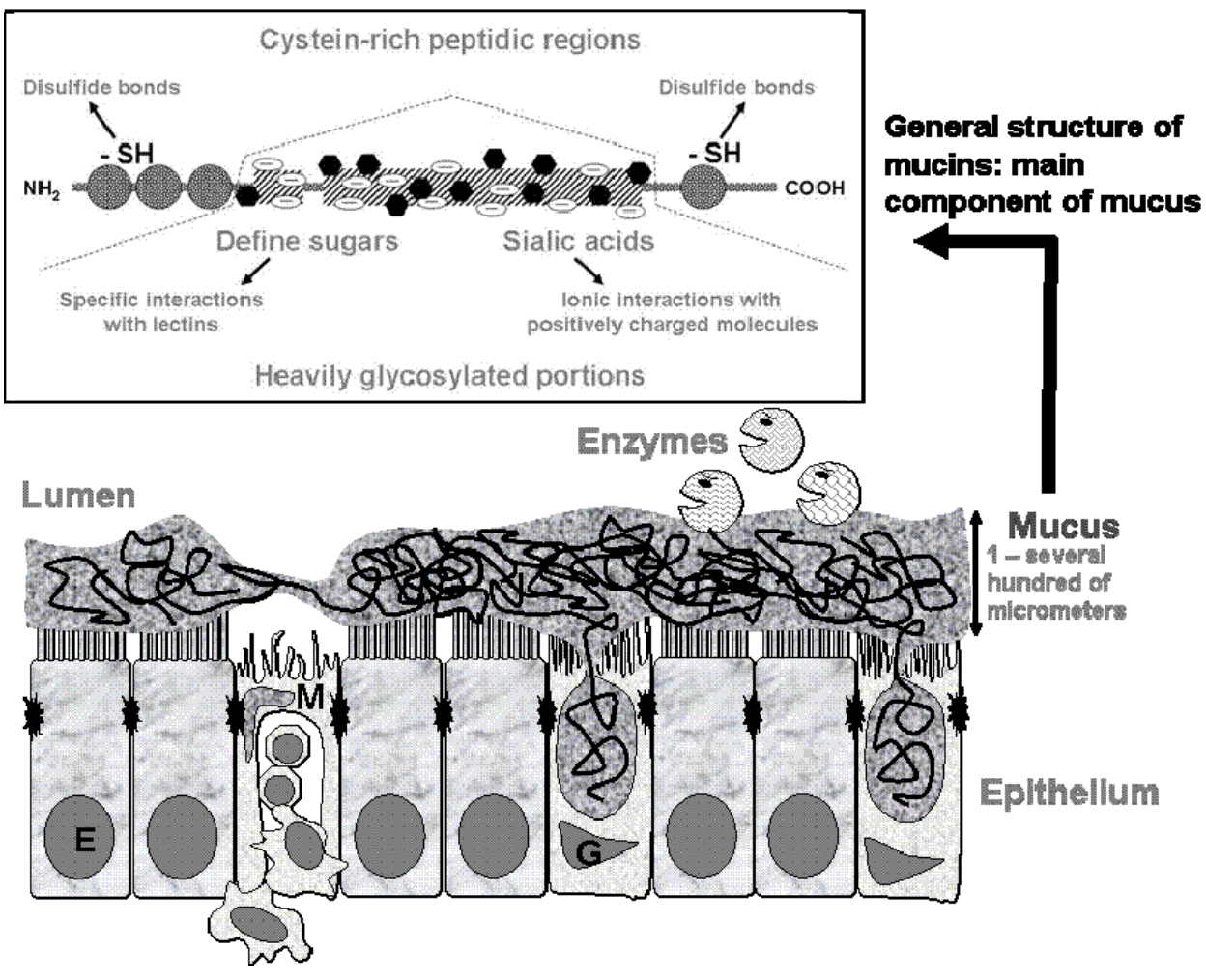

Figure 1: General structure of the gut mucosa and of mucins, main component of mucus. Cells in the epithelium are enterocytes (E), $M$ cells (M) and goblet cells ( $G)$. Inserted frame: The different relevant chemical groups involved in mucoadhesion are indicated as well as the different possible specific interactions that can be promoted with mucoadhesive drug delivery systems.

Thus, the close contact of the mucoadhesive drug delivery system with the mucus allows to increase of the drug concentration gradient at the surface of the epithelium which in turn favors drug absorption. The exact mechanism prevailing in mucoadhesion is still unknown but there are four well accepted theories that describe possible interactions involved in the mucoadhesion phenomenon (i.e:electronic, adsorption, wetting and diffusion theory) (Smart 2005, Ponchel and Irache 1998). All are based on very define types of interactions occurring between the mucoadhesive polymer and mucus glycoproteins which are composed of highly glycosylated proteins with a high content of sialic acid and thiol groups. (Cone 2009, Dodou et al. 2005). In the electronic theory of 
mucoadhesion, an electron transfer occurring between the mucus and the polymer leads to the formation of a double layer of electrical charges at the mucus/polymer interface. According to the adsorption theory, secondary forces such as hydrogen bonding and Van der Waals interactions are largely responsible for the interaction between the mucus and the adhesive polymers. The wetting theory applies mainly to adhesives in the liquid states in which the ability of the adhesive to swell and spread on the mucus layer depends on the interfacial energy between the two partners. Contact angle measurement can be used to predict interactions of the polymers with the mucus layer. Lower contact angle favors mucoadhesion. In the diffusion theory, interpenetration and physical entanglement of the mucoadhesive polymers with the mucus layer are the key factors for the adhesion process. Phenomena involved in this mucoadhesive mechanism depends on the molecular weight, the degree of cross-linking, the chain length, the flexibility and the spatial conformation of both the polymer and mucins found in the mucus (Smart 2005, Dodou et al. 2005). None of these theories gives by themselves a complete description of the mechanism of mucoadhesion. In general, the global phenomenon of mucoadhesion results from a combination of the four theories with a balance between them which depends on the nature of the mucoadhesive polymer and on the nature and composition of the mucus. In an actual scenario, it can be expected that the polymer gets wet and swells upon contact with the mucosal surface (wetting theory). Thereafter, noncovalent bonds including electrostatic and hydrophobic interactions are created at the mucus-polymer interface (electronic and adsorption theory). The nature and strength of these noncovalent bonds are greatly influenced by the chemical nature of the mucoadhesive polymer. Then, the polymer and mucin chains interpenetrate and entangle together (diffusion theory), to further reinforce the mucus-polymer interactions (Smart 2005).

Polymers should have some general characteristics which will enable them to function as mucoadhesive material (Table 1). The most widely investigated groups of mucoadhesives are hydrophilic macromolecules containing large number of hydrogen bond forming 
functional groups. The presence of hydroxyl, carboxyl or amine groups on the molecules are favorable to promote adhesion to mucosal surfaces. They are mostly called wet adhesives because they are activated by moistening and adhere to mucosal surfaces through non-specific interactions. Typical example includes polyacrylic acid, chitosan, sodium alginate and cellulose derivatives (Lowman and Peppas 1999). In general they are used as part of the components of a drug formulation designed to be administered by a mucosal route. In general, mucoadhesive polymers are included in conventional drug delivery formulations dedicated for mucosal routes of administration. At present, they are also widely considered for the development of technologies to be applied in nanomedicine including approaches for oral delivery of insulin.

\section{Mucoadhesive Nanoparticles for Drug Delivery Applications}

Polymeric nanoparticles are particles of less than $1 \mu \mathrm{m}$ in diameter that are prepared from natural or synthetic polymers (Hans and Lowman 2002). Nanoparticles offer numerous advantages over conventional drug delivery systems as smaller surface to volume ratio is their salient feature. The high specific surface area promotes a better interaction of a nanoparticle with the mucosal surface compared to conventional drug delivery systems such as gels, patches, tablets or capsules. However, from an experimental point of view, the current methods for mucoadhesion measurements do not provide direct evidence of the nature of polymer-mucin interactions, when applied to a nano-particulate system. The methods currently reported mostly exploit custom-made or modified equipments, and there is no universally accepted test method available yet. Although same hold true for any mucoadhesive material the situation appears more complex in the case of nanoparticles (Peppas and Huang 2004). This is the reason that so few comparisons of inter-group data appeared in the literature and that the definition of "good" mucoadhesives still remains a debatable issue. 
Table 1: Mucoadhesive polymers

\begin{tabular}{|c|c|c|c|c|}
\hline $\begin{array}{l}\text { Polymers and } \\
\text { functionalities }\end{array}$ & $\begin{array}{c}\text { Type of } \\
\text { interactions }\end{array}$ & Mode of interaction & Advantage & Disadvantage \\
\hline $\begin{array}{c}\text { Polyacrylic acid and } \\
\text { derivatives, } \\
\text { Carboxymethyl } \\
\text { Cellulose, } \\
\text { Alginate }\end{array}$ & Non-specific & $\begin{array}{l}\text { Carboxyl groups in these } \\
\text { polymers make H-bonding with } \\
\text { the hydroxyl groups in the } \\
\text { mucus layer }\end{array}$ & $\begin{array}{l}\text { Safe for oral delivery, } \\
\text { non-specific binding with } \\
\text { the intestinal epithelium, } \\
\text { inexpensive }\end{array}$ & Low in vivo efficacy \\
\hline $\begin{array}{l}\text { Chitosan and } \\
\text { derivatives, } \\
\text { amino dextran, } \\
\text { polydiaminomethyl } \\
\text { methacrylates }\end{array}$ & Non-specific & $\begin{array}{l}\text { Amino groups in the polymer } \\
\text { interact with the carboxyl group } \\
\text { residues in the mucus layer }\end{array}$ & $\begin{array}{c}\text { Safe for oral delivery, } \\
\text { electrostatic interaction with } \\
\text { mucus layer }\end{array}$ & $\begin{array}{l}\text { Poor solubility at physiological } \\
\text { conditions, strong interactions at } \\
\text { mucus layer, poor diffusion } \\
\text { property }\end{array}$ \\
\hline $\begin{array}{l}\text { PEG and their } \\
\text { derivatives }\end{array}$ & Non-specific & $\begin{array}{l}\text { Non-adhesive in nature, works } \\
\text { as adhesion promoter when } \\
\text { used along with a polymeric } \\
\text { system such as hydrogel }\end{array}$ & $\begin{array}{l}\text { Non-toxic, } \\
\text { no interaction with the } \\
\text { intestinal mucosal layer }\end{array}$ & $\begin{array}{l}\text { Excellent in vitro results, poor in } \\
\text { vivo performance }\end{array}$ \\
\hline Lectins & Highly specific & $\begin{array}{l}\text { Specifically binds to define } \\
\text { sugar residues of glycoproteins } \\
\text { found on the epithelial surfaces } \\
\text { and in mucus }\end{array}$ & $\begin{array}{l}\text { Specific binding, } \\
\text { non-toxic and non- } \\
\text { immunogenic }\end{array}$ & $\begin{array}{l}\text { Safety issues related to the } \\
\text { repeated use, cost factor etc. }\end{array}$ \\
\hline Thiols & Specific & $\begin{array}{l}\text { Make di-sulphide bonds with } \\
\text { mucus glycoproteins }\end{array}$ & $\begin{array}{l}\text { Specific binding mechanism, } \\
\text { ability of open epithelial } \\
\text { tight junctions }\end{array}$ & $\begin{array}{c}\text { Poor in vivo results, stability } \\
\text { issue }\end{array}$ \\
\hline
\end{tabular}

Specific functions enhancing mucoadhesive properties and principal characteristics of polymer interactions with mucus. 
In general, in vitro techniques currently used to evaluate mucoadhesion process involve destruction of the adhesive bond between the polymer and the mucus or tissue predominantly via application of mechanical force. It is obvious that many of these techniques are not applicable with nanoparticles. Therefore, it remains extremely difficult to investigate exact mechanism of nanoparticle mucoadhesion (Lowman and Peppas 1999). Despite these shortcomings, nanoparticles prepared from known mucoadhesive polymers are now widely investigated for developing novel drug delivery systems, especially for proteins and other large molecular weight drug molecules. A classification in four groups can be suggested based on the nature and properties of the material used to prepare them as explained below.

- Nanoparticles from conventional mucoadhesive polymers

- Mucus penetrating and diffusing nanoparticles

- Nanoparticles obtained by the functionalization of conventional mucoadhesive polymers.

- Nanoparticles becoming mucoadhesive by surface erosion.

\subsection{Nanoparticles from conventional mucoadhesive polymers}

Conventional or so-called first generation mucoadhesive nanoparticles are largely based on poly(acrylic acid) (PAA) including their crosslinked derivatives such as Carbopol, and Polycarbophil, chitosan (CS), alginate and cellulose derivatives . Mostly used as pharmaceutical excipients, these mucoadhesive polymers have also gained significance to formulate mucoadhesive nanoparticles.

Polyanionic polymers such as PAA operate mainly via non-covalent interactions with mucus including hydrogen bonding and Van der Waals forces (Table 1, Figure 2). Hydrogen bonding is expected to take place between carboxylic groups of the polymer chain and the mucin glycoproteins (Figure 1). 


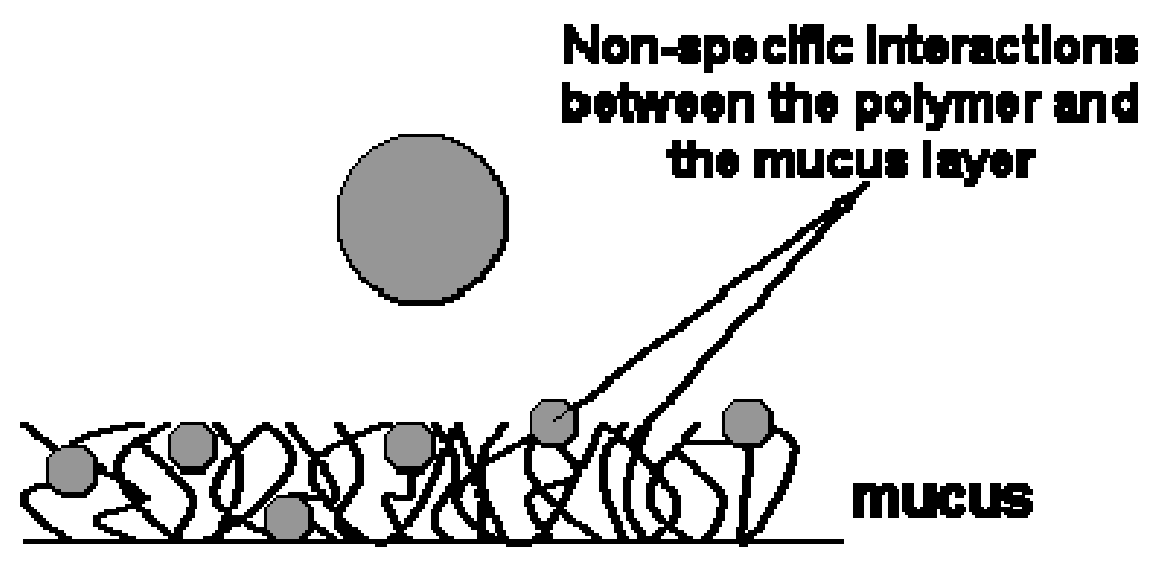

Figure 2: Interactions of conventional mucoadhesive nanoparticles with the gut mucosa. Nature of interactions is mainly nonspecific including non-ionic interactions between the nanoparticles and the mucus layer.

However, drug delivery systems formulated with PAA often failed to demonstrate strong mucoadhesive efficacy in vivo. This is attributed to the ionization of carboxylic groups of PAA at neutral/alkaline $\mathrm{pH}$ of the Gut. Indeed, PAA which has a pKa close to 5.0 gets easily ionized at the intestinal $\mathrm{pH}$ hence reducing hydrogen bonding interactions with the mucus layer. Another minor effect is attributed to the rigid structure of the polymers. In some examples, PAA was used in combination with poly(methacrylic acid) (PMAA) to obtain hydrogel nanoparticles adding several other values to the mucoadhesive formulation (Lowman and Peppas 1999).

Indeed, beside mucoadhesive properties, there are several other advantages of using hydrogels. Because of their high-water content, they constitute a 'protein-friendly' environment which is an excellent carrier matrix for such fragile molecules. Their preparations are based on organic solvent free methods and their loading with protein can be achieved by the manipulation of their physicochemical properties. One very interesting property in drug delivery is the ability of based hydrogels to respond to environmental stimuli such as variations of temperatures and of $\mathrm{pH}$. It is a unique 
advantage emphasizing developments of pharmaceutical formulations for the oral delivery of proteins. $\mathrm{pH}$ sensitive hydrogels contain ionizable groups for which the ionization status depends on the $\mathrm{pH}$ of the environment. Change in $\mathrm{pH}$ may ionized the acidic or basic functional groups creating electrostatic repulsion between polymer chains and in turn resulting in the swelling of the hydrogel (Lowman and Peppas 1999). For instance, encapsulated protein can be protected inside a PAA based hydrogel at acidic $\mathrm{pH}$ of the stomach ( $\mathrm{pH} \mathrm{1-2)} \mathrm{because} \mathrm{the} \mathrm{hydrogel} \mathrm{network} \mathrm{remains} \mathrm{collapsed} \mathrm{at} \mathrm{pH}$ below 5.0. As $\mathrm{pH}$ environment changes to alkaline conditions found in the gut, the hydrogel swells allowing the encapsulated protein to diffuse out of the hydrogel (Lowman et al. 1999). Other interesting features of mucoadhesive polymers composed of PAA are noteworthy regarding applications for oral delivery of protein and peptide drugs. PAA can act as calcium chelators resulting in calcium concentration depletion. This has two major benefits for oral delivery of protein and peptide drugs. First, depletion of calcium concentration in the gut inhibits proteolytic activity of the major proteases of the GIT, trypsine and chymotrypsine (Madsen and Peppas 1999). Protein and peptide drugs may be protected against degradation and their therapeutic activity may be protected. Secondly, it disturbs cell-cell adhesion by loosening the epithelial tight junctions hence drug permeation is improved (Staddon et.al.1995, Lowman and Peppas 1999). Regarding oral delivery of insulin, several types of mucoadhesive formulations were prepared considering polyanionic polymer-based nano and microparticles. Results for in vivo investigations showed a clear dose dependent effect occurring $2 \mathrm{~h}$ after oral administration of the insulin-containing formulation in both healthy and diabetic rats while the effect lasted at least $6 \mathrm{~h}$ (Lowman et al. 1999, Lowman and Peppas 2004).

Other conventional mucoadhesive polymers used to formulate mucoadhesive nanoparticles are polysaccharides including chitosan (CS) and alginate. 
Chitosan is a polycation prepared from chitin isolated from shrimps. Its strong mucoadhesive properties are explained by hydrogen and ionic bonds occurring between the negatively charged sialic acid residues of mucins and the positive charges of the amino groups of CS (Figure 1). Apart from the mucoadhesive properties, the polycationic form of chitosan is also able to open epithelial tight junctions enhancing the paracellular permeation of hydrophilic macromolecular drugs including insulin. It is noteworthy that the effect on the integrity of the epithelium and on the cell membranes of CS is much lower compared to that of known absorption enhancers (Illum 1998). CS then appears as a much safer permeation enhancing agent in addition to its mucoadhesive properties. Mucoadhesive nanoparticles can be formulated with chitosan by ionotropic gelation with tripolyphosphates which are negatively charge oligomers of phosphate ions. The formulations were proposed as drug delivery systems for oral administration of insulin. Efficacy of CS-nanoparticles to enhance oral absorption of pharmacologically active insulin was investigated by monitoring the glycemia of alloxan-induced diabetic rats. Results indicated that the CS-nanoparticles enhanced the intestinal absorption of insulin to a greater extent in comparison with an aqueous solution of CS. CSnanoparticles increased remarkably the relative bioavailability of insulin compared to a subcutaneous injection of an insulin solution. While the glycemia was reduced over a period of $15 \mathrm{hrs}$ after administration of $21 \mathrm{IU} / \mathrm{kg}$ insulin in the CS-NPs, the average relative pharmacological bioavailability was up to $14.9 \%$ (Pan et al. 2002). By increasing the dose in insulin in the CS-nanoparticles up to $100 \mathrm{IU} / \mathrm{kg}$, the glycemia was markedly reduced at 10 hours post-administration. Then, an interesting prolonged pharmacological effect lasted for up to 24 hours (Pan et.al. 2002). In another work, CS nanoparticles were prepared with hydroxypropyl methyl cellulose phthalate (HPMCP) which is a $\mathrm{pH}$ sensitive and gastroresistant polymer. The mucoadhesion of the nanoparticles was 2 to 4 times superior to that of the corresponding CS-tripolyphosphate nanoparticles. Penetration of the nanoparticles in the mucosa was improved compared to that of the 
reference CS-tripolyphosphate nanoparticles. The hypoglycemic effect of insulin obtained after peroral administration of the CS/HPMCP nanoparticles was also markedly increased. It was more than 9.8 and 2.8 -folds compared to that produced by oral administration of a solution of insulin and of insulin-loaded CS-tripolyphosphate nanoparticles, respectively (Makhlof et al. 2010). In an earlier work, HPMCP was considered as a coating material for CS capsules to design a delivery system for insulin to the colon (Tozaki et.al. 1997). A significant improvement of the oral bioavailability of insulin in rats was reported after oral administration of a dose of 20 IU. While the oral bioavailability compared to the intravenous route was $5.3 \%$, the glycemia dropped down 6 hours after oral administration and the effect lasted for $24 \mathrm{hrs}$ thereafter. Improvement of the performance of CS-nanoparticles can be obtained by modifying the chemical structure of the polysaccharide on its amino groups. For instance, trimethylation was aimed to improve the solubility of $\mathrm{CS}$ in aqueous solutions at $\mathrm{pH}$ approaching neutral values. This has dual advantages as the trimethyl chitosan (TMC) is protonated at $\mathrm{pH}$ above 6.5. The polymer chains are fully soluble in aqueous media of $\mathrm{pH}$ above 6.5 which is in contrast with that of CS which tend to aggregate in these media (Thanou et.al. 2001). The TMC mucoadhesion is improved at the intestinal $\mathrm{pH}$ compared to that of CS. The second advantage concerns the effect of CS on the opening of the tight junctions which is very important to facilitate the paracellular transport of hydrophilic compounds including insulin. As only the protonated CS occurring at $\mathrm{pH}$ below 6.5 can trigger the opening of the tight junctions, TMC providing with a protonated form at $\mathrm{pH}$ above 6.5 found in the gut is suitable to improve paracellular permeability of the intestinal epithelium (Thanou et al. 2001). Promising results were obtained from TMC nanoparticles in experiments based on in vitro cell cultures of the cell monolayer model of Caco 2 (Thanou et al. 2001). Apart from nanoparticles obtained from TMC, other derivatives of CS were used to develop nano and microparticles for oral delivery of proteins. Typical examples included phthalate, succinate, PEGylated derivatives of CS. 
Alginate is another polysaccharide use to formulate mucoadhesive nanoparticles for oral delivery of insulin. Most methods of preparation include an ionotropic pre-gelation of the alginate with divalent calcium followed by complexation with a polyelectrolyte. Calcium crosslinked alginate particles can protect peptides from gastric degradation by virtue of pH dependent release mechanism (Tønnesen and Karlsen 2002, Sarmento et al. 2007). After oral administration to rats, the superiority of alginate nanoparticles to improve bioactivity and absorption of insulin was attributed to the mucoadhesive properties of the formulation and to an improvement of the internalization of the peptide within the intestinal mucosa (Sarmento et al. 2007).

\subsection{Mucus Penetrating and Diffusing Nanoparticles}

Efficacy of conventional mucoadhesive nanoparticles can be compromised by the interaction of the drug delivery device with soluble mucins found in the lumen of the GIT (Peppas and Huang 2004). Therefore, they are removed quickly from the intestine before they can reach the epithelium where drug absorption takes place. In order to counterbalance this effect, it was suggested to develop mucoadhesive formulations that bind specifically to the mucus layer. The rational behind this approach was to improve the inter-diffusion and inter-penetration of polymeric system in the mucosal barrier by reducing the interfacial energy and glass transition temperature of the parent polymer composing the nanoparticles. To this purpose, adhesion promoters were introduced in conventional mucoadhesive polymers. A typical example of adhesion promoter is composed of a poly(ethylene glycol) (PEG) containing macromer which can be easily incorporated in the mucoadhesive PAA/PMAA copolymer by copolymerization. Resultant material possesses good chain mobility/flexibility in order to diffuse across the mucosal layer and avoid soluble mucin binding through a steric effect. PEG was also used to coat poly(lactide-co-glycolide) (PLGA) nanoparticles and poly(sebacic acid) nanoparticles (Lai et al. 2009) (Figure 3). 


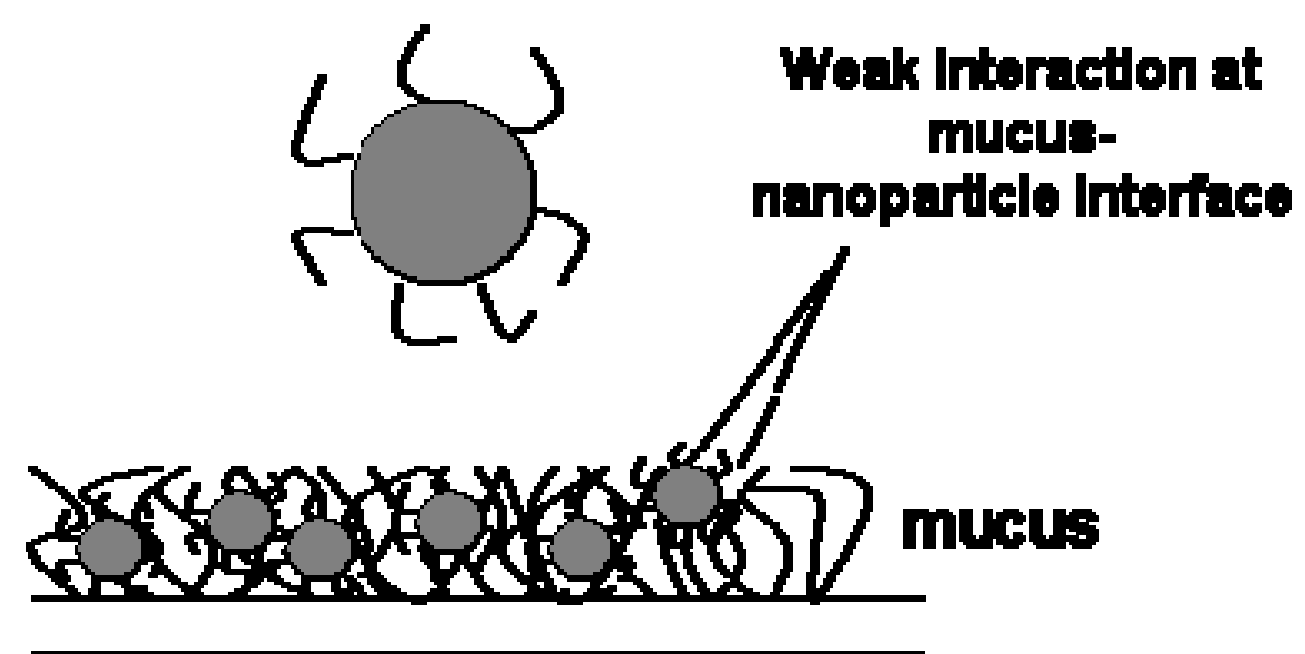

Figure 3: Interactions of mucus penetrating and diffusing nanoparticles with gut mucosa. The nanoparticles are coated with polymer chains enabling the nanoparticles to diffuse through the mucus layer avoiding strong interactions with mucins.

The diffusivity of the PEG-PLGA nanoparticle across the mucus was only reduced by 4 and 12 times respectively compared to the diffusivity evaluated for the same nanoparticles in water. In comparison, the corresponding non-coated nanoparticles were 3300 folds lower in mucus than in water. Results of diffusivity measurements suggest that surface modification of the nanoparticles can greatly improve diffusivity of the nanoparticles in the mucus layer overcoming the soluble mucus barrier. A new generation of mucoadhesive nanoparticles is about to emerge while it is now necessary to combine these findings with developments of nanoparticles showing tailored drug release profile (Lai et al. 2009).

As alternative polymers to PEG, polysaccharides are other interesting material to be used to decorate polymeric nanoparticles aiming to improve their mucoadhesion properties. This approach was mainly developed in a series of core-corona nanoparticles composed of a poly(isobutyl cyanoacrylate)hydrophobic core surrounded by an hydrophilic corona composed of polysaccharides such as CS and dextran (Bertholon et al. 2006, BravoOsuna et al. 2007). Large amounts of the CS-coated nanoparticles were entrapped in the 
mucus layer even at low nanoparticles concentration. With dextran-coated nanoparticles, the mucoadhesive phenomena was non saturable in contrast to what was observed with CS decorated nanoparticles. This finding agreed with the fact that CS may adhere to mucus components through electrostatic interactions where the number of sites is finite giving saturable phenomenon. It can be concluded that that bioadhesive properties of nanoparticles can be finely tuned by modifying surface properties of the nanoparticles.

\subsection{Functionalization of nanoparticles made of conventional mucoadhesive polymers}

Highly specific mucoadhesive properties can be given to the nanoparticles by decorating them with defined motives (Figure 4). The rational behind this is to promote adhesion of nanoparticle drug delivery system on a define portion of the intestine where absorption sites of the drug are located. Improvements of mucoadhesive properties are obtained by modifying surface properties of nanoparticles. This can be achieved by chemical or physical coupling methods of ligands interacting with mucus on already prepared nanoparticles or on the polymer prior to the synthesis of the nanoparticles (Lehr 2000, Wood et al. 2008).

Lectins are typical examples of relevant ligands. They are non-immunologic glycoproteins recognizing very define sugar molecules (Lehr 2000). They can bind to glycosylated membrane components overlaying the GIT mucosa surface. For instance, the wheat germ agglutinin (WGA) extracted from plants can bind to $\mathrm{N}$-acetylglucosamine and sialic acid residues found in the GIT. In vitro, it shows the highest binding rate to intestinal cells of human origin compared to lectins from other plant sources. Interesting studies were reported in the literature considering the use of lectins to improve specificity of mucoadhesion of nanoparticles in the GIT (Ponchel and Irache 1998, Gao 2006). Only a limited number of works have considered application of the approach on biodegradable nanoparticles and were aimed to improve oral delivery of peptides. 


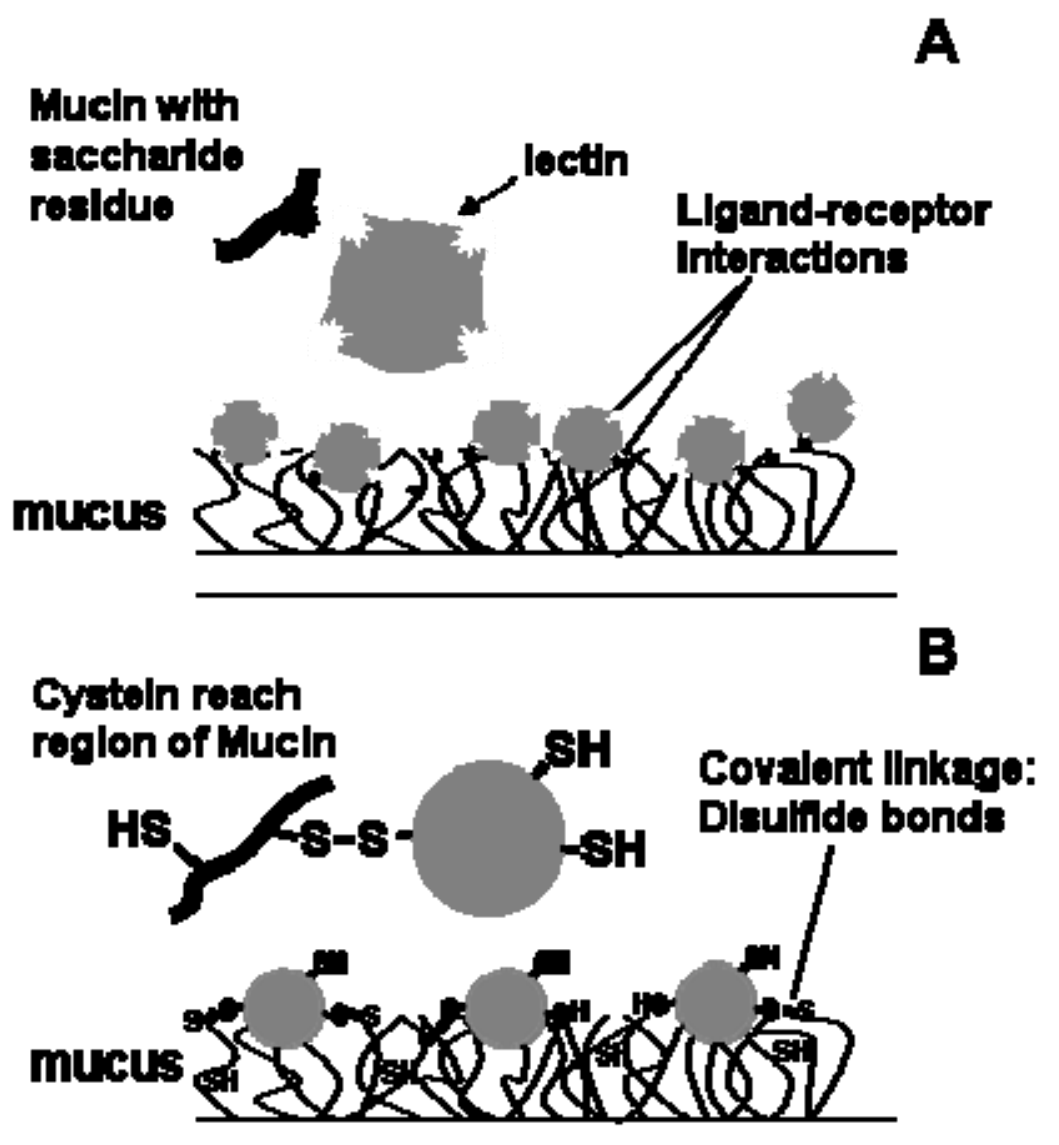

Figure 4: Interactions of functionalized nanoparticles with the gut mucosa. Nanoparticles can be functionalized with lectins (A) or thiol (B) groups promoting specific interactions with define sugar residues of mucins or thiol groups of the cysteinrich region of mucin respectively.

A lectin modified PLGA nanoparticles loaded with a synthetic pentapeptide used in immunomodulation therapy, the thymopentin, showed an enhanced interactions with pig mucus by 1.8 to 4.2 folds compared with interactions measured in same conditions with the unmodified PLGA nanoparticles (Yin et.al. 2006). In another example considering oral insulin delivery, WGA was conjugated to alginate microparticles (Kim et.al. 2005). The hypoglycemic effect of the WGA-decorated particles was the most pronounced. However, it was not demonstrated that the effect was actually due to an effective 
prolonged residence time in the GIT tract thanks to an improvement of the mucoadhesiveness of the particles. In another work, the mucoadhesive nature of PMAAgrafted-ethylene glycol hydrogel particles was enhanced with WGA thanks to specific interactions with carbohydrates found on mucins (Wood et al. 2008) (Figure 1 and 4A). Improved mucoadhesive properties was accompanied by enhanced absorption of insulin as evaluated in vitro suggesting that the WGA decorated PMAA grafted ethylene glycol holds great promise as an oral formulation for the delivery of insulin improving diabetes treatments (Wood et al. 2008).

Another approach improving mucoadhesion with mucus is based on the use of thiol (sulphide) groups of the cystein rich region of mucins to promote strong and specific interactions with nanoparticles (Figure 1 and 4B). In this aim, the nanoparticle surface is decorated with thiol groups. The rational is to enhance adhesion of nanoparticles on the mucus by formation of covalent disulfide bonds between thiol groups available on the surfaces of the two partners. The strategy was initially suggested by the group of Bernkop-Schnurch. (Bernkop-Schnürch et.al. 2003). It was widely used for the development of drug delivery platforms for oral administration of hydrophilic macromolecules such as insulin, heparin and salmon calcitonin (Bernkop-Schnürch et.al. 2003). Addition of thiol groups can induce transient and reversible increase of the permeability of the intestinal epithelium thanks to specific interactions with proteins of the tight junctions. This interesting effect was recognized to increase transport of drugs by the paracellular pathway and could be applied to improve oral bioavailability of protein and peptide drugs.

Thiol groups can be introduced on the surface of nanoparticles by coupling molecules such as cysteine, thioglycolic acid, cysteamine on mucoadhesive polymers like PAA, CS, alginate, carboxymethyl cellulose. Standard conjugation methods are suitable and the approach was recently applied on nanoparticles designed for oral delivery of insulin. In 
general, results showed improvement of both the efficacy of the thiol modified drug delivery system and of the mucoadhesion. For instance, nanoparticles containing PAAcysteine increased 2.3 folds the area under the curve (AUC) of insulin administered orally to diabetic rats compared with the efficacy given by the corresponding non modified PAA formulation (Deutel et al. 2008). Although the incorporation of cystein in one of the nanoparticle formulations improved the efficacy of insulin delivery, the relative bioavailability of the peptide remained extremely low $(0.2$ and 0.1 respectively for PAACysteine and PAA systems) compared to the subcutaneous injection of insulin. In another example, thiol modification achieved by grafting of cystein on surface of poly(methacrylic acid)-chitosan-poly(ethylene glycol) (PCP) particles (diameter around 1 $\mu \mathrm{m})$ significantly improved transport of insulin across Caco 2 cells and mucoadhesion on excised rat intestinal tissue compared with the unmodified PCP particles (Sajeesh et.al. 2010). In vivo, the thiol modified PCP particles were more effective in reducing blood glucose level in diabetic rats. The benefit brought by the introduction of thiol groups on the drug delivery systems to promote mucoadhesion and efficacy of oral administration of insulin was further confirmed in a recent work considering TMC nanoparticles. The introduction of cysteine residues in the structure of the TMC nanoparticles improved mucoadhesive properties by 4.7 folds compared with the non-modified TMC nanoparticles. This improvement correlated well with the increase of insulin transport through the rat intestine which ranged from 1.7 to 2.6 for the TMC nanoparticles and from 3.3 to 11.7 folds with the TMC-cysteine nanoparticles (Yin et al. 2009). Further work is required to elucidate whether the improvement of the mucoadhesion was the only mechanism responsible for the increase of the oral efficacy of insulin delivered by the thiol modified nanoparticles or whether the permeation of the epithelium was increased because of the presence of the thiol groups. It is probable that both mechanisms have contributed to the enhanced delivery of insulin observed with the thiol modified particles but the part of each mechanism in this contribution remained to be determined. 


\subsection{Nanoparticles becoming mucoadhesive by surface erosion.}

In general, hard bioerodible thermoplastic polymers such as polyesters and polyanhydrides are not mucoadhesives. However, they were found interesting potential mucoadhesive polymers to formulate nanoparticles for oral drug delivery applications. Actually, polymers like poly(fumaric-co-sebacic) (PFASA) anhydride can rapidly degrade in contact with aqueous media relealing mucoadhesive properties thanks to the appearance of carboxylic acid groups resulted from the hydrolytic cleavage of the anhydride bond (Figure 5).

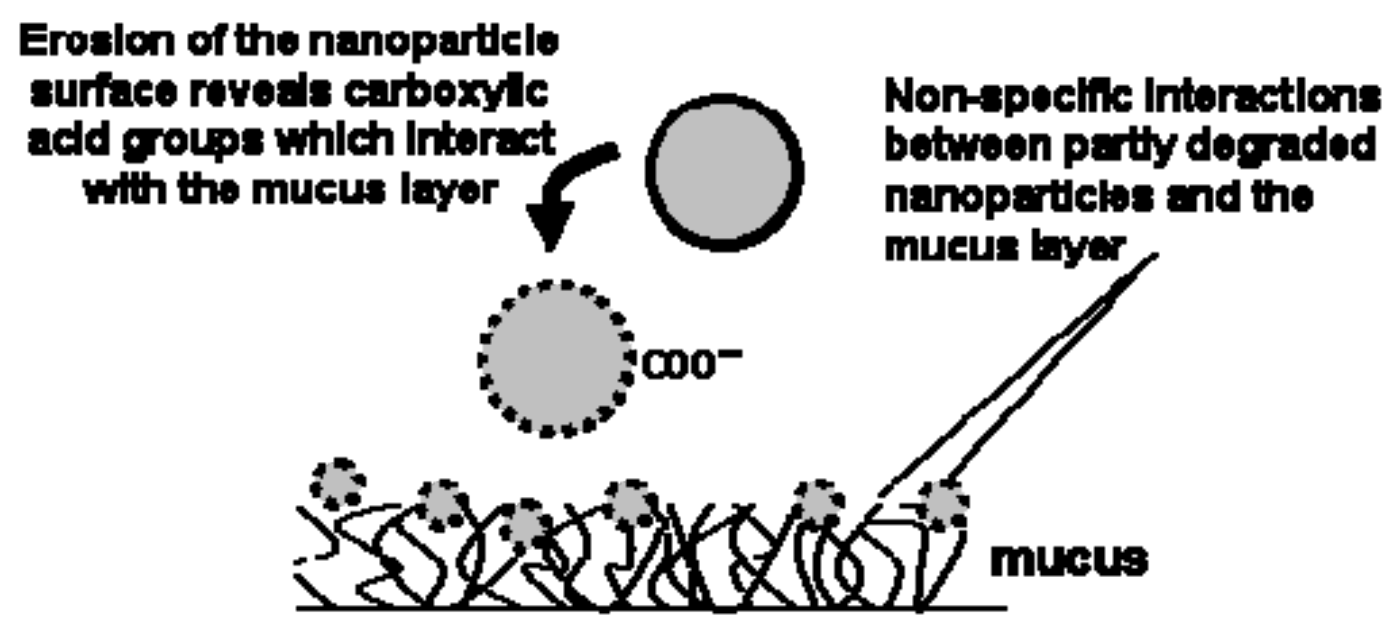

Figure 5: Interactions of surface eroding nanoparticles with the gut mucosa. Typical example are poly(anhydride)-coated nanoparticles that reveal carboxylic group during hydrolysis.

The increase of the number of carboxylic acid groups appearing at the nanoparticle surface during degradation enhances the ability of the polymer to form hydrogen bonds with mucus and epithelial cell glycoproteins promoting the adherence of the nanoparticles on the mucosa. Mucoadhesive properties shown by such polyanhydride nanoparticles on small intestinal tissue of rats appeared stronger than those of all other nanoparticles made of other polymers (Chickering et al. 1996). In vivo, the transit of the 
nanoparticles is delayed while the relative bioavailability of a drug can be improved. It was proposed that formulations including such hydrophobic polymers can be retained in the GIT and possibly increase intestinal absorption of drugs (Chickering et al. 1996). The prolonged residence time of particles in the GIT may increase the chance of particles to be taken up by enterocytes which were observed to cross the mucosal epithelium and the follicle-associated epithelium covering the lymphatic elements of Peyer's patches (Mathiowitz et.al. 1997). Particles of p(FASA) with a diameter around $5 \mu \mathrm{m}$ were applied to the oral delivery of insulin on type 1 diabetic rats and dogs. Different bioavailabilities of insulin were observed depending on the type of animals and on the administration schedule In diabetic rats, the fed double dose experiment produced a relative bioavailability of $23.3 \%$ while the fed single dose experiment produced a bioavailability of only $5.5 \pm 1.7 \%$ (Furtado 2008). In fasted diabetic dogs, the relative bioavailability was $5.5 \pm 3.4 \%$. Oral delivery of active zinc insulin was also achieved with another type of particles formulated with a combination of PLGA and oligomers of fumaric acid as polyanhydrides. The formulation was able to control plasma glucose levels when faced with a simultaneously administered glucose challenge. The efficacy was $11.4 \%$ of that of an intraperitoneal delivery of zinc insulin. The rational behind the use of these polymers revealing mucoadhesive properties upon degradation is a tricky approach allowing to immobilize the drug delivery device in the mucus releasing high local concentration of drugs close to absorption sites of the intestinal epithelium.

\section{Conclusion}

Bioadhesive polymers are promising materials in designing drug delivery systems for mucosal barriers. However, oral delivery of proteins and peptides still remains a bottleneck. Attempts to improve oral delivery of proteins and peptides have been quite unsuccessful so far in obtaining satisfactory and consistent results. For instance, a therapeutic protein like insulin used in the treatment of diabetes needs to be administered 
on a daily basis with a very precise and robust delivery method able to regulate the pharmacological response at an optimum level. In this review, it was shown that technologies from nanomedicines can improve oral insulin delivery just by improving mucoadhesion of the nanoparticles. Although still insufficient, further improvements can be expected from a better optimization of the mucoadhesive properties and adding to the delivery systems other functionalities including permeation enhancing properties. A lot of work remained to be done but technologies from nanomedicine bring new hopes to succeed in obtaining an oral treatment of insulin available to diabetic patients. 


\section{Summary Points}

- Penetration of drugs in mucus is required to reach absorption sites stranded on the gut epithelium

- Mucoadhesion is a complex phenomenon that results from the combination of 4 main mechanisms.

- Certain polymers exhibit mucoadhesion phenomenon.

- Mucoadhesive nanoparticles promote oral delivery efficacy of active insulin

- Mucoadhesion should take place in the mucus layer and not with soluble mucins of the lumen

- Mucus penetrating/diffusing nanoparticles showed better mucoadhesive performance

- Mucoadhesion of polymeric nanoparticles can be improved by specific modification approach

- Thiol modification improves mucoadhesion of nanomedicine by formation of disulfide bonds with cystein rich region of mucins and confers enhancing permeation properties promoting drug absorption through the paracellular pathway.

- Mucoadhesive technologies from nanomedicine bring true hope for the development of a treatment of diabetes based on oral insulino-therapy.

\section{Key Facts}

- Key facts on oral delivery of insulin: Oral delivery of insulin is facing many challenges due to the physiology of the gastrointestinal tract.

- Key facts on nanomedicine technologies: Technologies from nanomedicines provide opportunities to improve drug delivery efficacy and targeting. It can make oral insulino-therapy a reality to patients in near future.

- Key facts on mucoadhesion: Mucoadhesion promotes interactions of exogenous particles with mucus overlaying the epithelial cells in the gastrointestinal tract. This can help mucoadhesive nanoparticles to reach absorption sites found on the gut epithelium.

- Key facts on designing mucoadhesive nanoparticles: Mucoadhesiveness of nanoparticles depends on nanoparticle surface properties which in turn govern the different types and strength of interactions promoted between particles and mucus components. Major difficulty here is to find a proper balance between strength of interactions allowing adequate adherence of nanoparticles onto the mucus and at the same time allowing particles to penetrate and diffuse through the mucus layer to access enterocytes. Modulating surface properties of nanoparticle delivery system seems to be the key in improving their efficacy towards oral insulin delivery. 


\section{Definitions}

Copolymer: polymer made of at least two monomers

Copolymerization: Method of synthesis of copolymer based on the polymerization of at least two types of monomer together.

Homopolymer: Macromolecule formed from one type of repeated unit

Hydrogel: polymeric material formed by a tridimensional network of polymer chains swelled with water.

Macromer: monomer of high molecular weight.

Monomer: generally small chemical molecule which can polymerize meaning that they can be react together to form long polymer chains

Mucin: glycoproteins found in mucus

Mucoadhesion: adhesion to mucosa.

Mucus: hydrogel material composed of mucins found on the top of the epithelium in mucosa.

Nanoparticles: small polymer nanoparticles characterized by a diameter ranging from 1 to $1000 \mathrm{~nm}$. Most nanoparticles designed as drug delivery systems have size ranging from 70 to $300 \mathrm{~nm}$ or around $1 \mu \mathrm{m}$ for the largest.

Polymer: general term to design macromolecule resulted from the self addition of the same monomer unit or by the addition of several types of monomer units. 


\section{References}

Arbit, E. 2004. The physiological rationale for oral insulin administration. Diabetes Technol. Ther. 6: 510-517.

Bernkop-Schnurch, A. and C.E. Kast, and D. Guggi. 2003. Permeation enhancing polymers in oral delivery of hydrophilic macromolecules: thiomer/GSH systems. J. Control. Release 93: 95-103.

Bertholon, I. and G. Ponchel, D. Labarre, P. Couvreur, and C. Vauthier 2006. Bioadhesive properties of poly(alkylcyanoacrylate) nanoparticles coated with polysaccharide. J Nanosci. Nanotech. 6: 1-8.

Bravo-Osuna, I. and C. Vauthier, A. Farabollini, G.F. Palmieri, and G. Ponchel. 2007. Mucoadhesion mechanism of chitosan and thiolated chitosan-poly(isobutyl cyanoacrylate) core-shell nanoparticles. Biomaterials. 28: 2233-2243.

Chickering, D. and J. Jacob, and E. Mathiowitz. 1996. Poly(fumaric-co-sebacic) microspheres as oral drug delivery systems. Biotechnol. Bioeng. 52: 96-101

Cone, R.A. 2009. Barrier properties of mucus. Adv. Drug Deliver. Rev. 61: 75-85

Deutel, B. and M. Greindl, M. Thaurerm, and A. Bernkop-Schnürch. 2008. Novel insulin thiomer nanoparticles: in vivo evaluation of an oral drug delivery system. Biomacromolecules. 9: 278-285

Dodou, D. and P. Breedveld, and P.A. Wieringa. 2005. Mucoadhesives in the gastrointestinal tract: revisiting the literature for novel applications. Eur. J. Pharm. Biopharm. 60: 1-16.

Furtado, S. and D. Abramson, R. Burrill, G. Olivier, C. Gourd, E. Bubbers, and E. Mathiowitz. 2008. Oral delivery of insulin loaded poly(fumaric-co-sebacic) anhydride microspheres . Int. J. Pharm. 347: 149-155.

Gao, X. and W. Tao, W. Lu, Q. Zhang, Y. Zhang, X. Jiang, and S. Fu, S. 2006. Lectinconjugated PEG-PLA nanoparticles: Preparation and brain delivery after intranasal administration. Biomaterials 27: 3482-3490.

Hans, M.L. and A.M. Lowman. 2002. Biodegradable nanoparticles for drug delivery and targeting. Curr. Opin. Solid St M. 6: 319-327

Illum, L. 1998. Chitosan and its use as a pharmaceutical excipient. Pharm. Res.15: 13261231.

Kim, B-Y. and J.H. Jeong, K. Park, and J.D. Kim. 2005. Bioadhesive interaction and hypoglycemic effect of insulin-loaded lectin-microparticle conjugates in oral insulin delivery system. J. Control. Release 102: 525-538.

Lai, S.K. and Y.Y. Wang, and J. Hanes. 2009. Mucus-penetrating nanoparticles for drug and gene delivery to mucosal tissues Adv. Drug Deliver. Rev. 27: 158-171

Lee, V.H.L. and A. Yamamoto. 1989. A Penetration and enzymatic barriers to peptide and protein absorption. Adv. Drug Deliver. Rev 4: 171-207. 
Lehr, C.M. 2000. Lectin-mediated drug delivery: The second generation of bioadhesives. J. Control. Release. 65: 19-29.

Lowman, A. M. and M. Morishita, M. Kajita, T. Nagai, and N.A. Peppas. 1999. Oral delivery of insulin using pH-responsive complexation gels. J. Pharm. Sci. 88, 933937.

Lowman, A.M. and N.A. Peppas. Hydrogels. pp- 397-418. In: E. Mathiowitz [Ed] 1999 Enclyopedia of Controlled Drug Delivery- Vol 1, Wiley, New York, USA

Madsen, F. and N.A. Peppas. 1999. Complexation graft copolymer networks: swelling properties, calcium binding and proteolytic enzyme inhibition. Biomaterials. 20: 17011708

Makhlof, A. Tozuka, Y. and Takeuchi H. 2010. Design and evaluation of novel pHsensitive chitosan nanoparticles for oral insulin delivery. Eur. J. Pharm. Sci. (In Press)

Mathiowitz, E. and J.S. Jacob, Y.S. Jong, C.P. Carino, D.E. Chickering, P. Chaturvedi, C.A. Santos, K. Vijayaraghavan, S. Montgomery, M. Bassett M., and C. Morell. 1997. Biologically erodable microspheres as potential oral drug delivery systems. Nature 386: 410-414.

Pan, Y. and Y.J. Li, H.Y. Zhao, J.M. Zheng, H. Xu, G. Wei, J.S. Hao, and F.D. Cui. 2002. Bioadhesive polysaccharide in protein delivery system: chitosan nanoparticles improve the intestinal absorption of insulin in vivo. Int. J. Pharm. 249: 139-47.

Peppas, N.A. and Y. Huang. 2004. Nanoscale technology of mucoadhesive interactions. Adv. Drug Deliver. Rev. 56: 1675-1687

Ponchel, G. and J. Irache. 1998. Specific and non-specific bioadhesive particulate systems for oral delivery to the gastrointestinal tract. Adv. Drug Deliver. Rev. 34: $191-219$

Ramesan, M.R. and C.P. Sharma. 2009. Challenges and advances in nanoparticle-based oral insulin delivery Expert Rev. Med. Devices, 6, 665-676.

Sajeesh, S. and C. Vauthier, C. Gueutin, G. Ponchel, and C.P. Sharma. 2010. Thiol functionalized polymethacrylic acid based hydrogel microparticles for oral insulin delivery. Acta Biomater. 06: 3072-3080.

Sarmento, B. And A. Ribeiro, F. Veiga, P. Sampaio, R. Neufeld, and D. Ferreira. 2007. Alginate/chitosan nanoparticles are effective for oral insulin delivery. Pharmceu. Res. 24: 2198-206.

Smart, J.D. 2005. The basics and underlying mechanisms of mucoadhesion Adv. Drug Deliver. Rev. 57: 1556-1568

Staddon, J.M. and K. Herrenknecht, C. Smales, C. and L.L. Rubin. Evidence that tyrosine phosphorylation may increase tight junction permeability. J. Cell Sci. 108: 609-619

Thanou, M. and J.C. Verhoef, and H.E. Junginger. 2001. Chitosan and its derivatives as intestinal absorption enhancers. Adv. Drug Deliv. Rev. 50: S91-S101

Tozaki, H. and J. Komoike, C. Tada, T. Maruyama, A. Terabe, T. Suzuki, A. Yamamoto, and S. Muranishi. 1997. Chitosan capsules for colon drug delivery: improvement of insulin absorption from rat colon. J. Pharm. Sci. 86: 1016-1021 
Tønnesen, H. H. and J. Karlsen. 2002. Alginate in drug delivery systems. Drug Dev. Ind. Pharm. 28: 621-630

Wood, K.M. and G.M. Stone, and N.A. Peppas. 2008. Wheat germ agglutinin functionalized complexation hydrogels for oral insulin delivery. Biomacromolecules. 9: 1293-1298

Yin, L. and J. Ding, C. He, L. Cui, C. Tang, and C. Yin. 2009. Drug permeability and mucoadhesion properties of thiolated trimethyl chitosan nanoparticles in oral insulin delivery. Biomaterials. 30: 5691-5700.

Yin, Y. and D. Chen, M. Qiao, Z. Lu, and H. Hu. 2006. Preparation and evaluation of lectin-conjugated PLGA nanoparticles for oral delivery of thymopentin. J. Control. Release. 116: 337-345. 